\title{
Exact Outage Probability of Dual-Hop CSI-Assisted AF Relaying over Nakagami- $m$ Fading Channels
}

\author{
Minghua Xia, Yik-Chung Wu, and Sonia Aïssa, Senior Member, IEEE
}

\begin{abstract}
In this paper, considering dual-hop channel state information (CSI)-assisted amplify-and-forward (AF) relaying over Nakagami- $m$ fading channels, the cumulative distribution function (CDF) of the endto-end signal-to-noise ratio (SNR) is derived. In particular, when the fading shape factors $m_{1}$ and $m_{2}$ at consecutive hops take non-integer values, the bivariate $H$-function and $G$-function are exploited to obtain an exact analytical expression for the CDF. The obtained CDF is then applied to evaluate the outage performance of the system under study. The analytical results of outage probability coincide exactly with MonteCarlo simulation results and outperform the previously reported upper bounds in the low and medium SNR regions.
\end{abstract}

Index Terms-Amplify-and-forward (AF) relaying, channel state information (CSI)-assisted, distribution function, dual-hop, Nakagami- $m$ fading.

\section{INTRODUCTION}

$\mathbf{F}$ OR dual-hop amplify-and-forward (AF) relaying systems, the gain of relaying node aims to invert the first-hop (source-torelay) channel and is determined by the channel state information (CSI) of the first hop. According to the amount of CSI obtained at the relay, there are three different $\mathrm{AF}$ schemes that can be implemented: blind, semi-blind, and CSI-assisted relaying. Among them, the theoretical analysis of CSI-assisted relaying is extremely important since this scheme characterizes the best performance.

Let $\gamma_{1}$ and $\gamma_{2}$ be the instantaneous signal-to-noise ratios (SNRs) of two consecutive hops, respectively, the end-to-end SNR of CSIassisted relaying can be derived as $\gamma_{\text {end }}=\frac{\gamma_{1} \gamma_{2}}{\gamma_{1}+\gamma_{2}+1}$ [1]. However, due to the existence of the unity in the denominator, which corresponds to the additive white Gaussian noise (AWGN) at the relay, exact performance analysis was usually considered to be intractable over arbitrary Nakagami- $m$ fading channels [1]. For the special case with integer Nakagami- $m$ fading channels, a large number of performance analyses were reported (see [2], [3] and references therein). However, the propagation environments where the Nakagami fading parameter takes non-integer values are very common in practice, such as micro-cellular scenarios with strong specular components and land mobile satellite channels. Therefore, exact performance analysis of CSI-assisted relaying over arbitrary Nakagami- $m$ fading is of great practical importance, but it still remains an open problem due to potential mathematical difficulty [4].

Copyright (c) 2012 IEEE. Personal use of this material is permitted. However, permission to use this material for any other purposes must be obtained from the IEEE by sending a request to pubs-permissions@ieee.org.

Manuscript received August 11, 2011; revised February 8, 2012, May 28, 2012 and June 20, 2012; accepted June 20, 2012. The associate editor coordinating the review of this manuscript and approving it for publication was Prof. Maja Bystrom. The work was supported in part by the Hong Kong University Seed Funding Programme under Project 201111159122, and by King Abdullah University of Science and Technology (KAUST).

M. Xia is with the Division of Physical Sciences and Engineering, KAUST, Thuwal, Saudi Arabia (e-mail: minghua.xia@ieee.org).

Y.-C. Wu is with the Department of Electrical and Electronic Engineering, The University of Hong Kong, Hong Kong (e-mail: ycwu@eee.hku.hk).

S. Aïssa is with INRS, University of Quebec, Montreal, QC, Canada and with KAUST, Thuwal, Saudi Arabia (e-mail: sonia.aissa@ieee.org).

Digital Object Identifier ***
For the CSI-assisted relaying over arbitrary Nakagami- $m$ fading channels, in order to obtain analytical performance metrics, two upper bounds for $\gamma_{\text {end }}$ are widely exploited in the literature. Specifically, the upper bounds are $\gamma_{\text {end }}<\frac{\gamma_{1} \gamma_{2}}{\gamma_{1}+\gamma_{2}}$ and $\gamma_{\text {end }}<\min \left\{\gamma_{1}, \gamma_{2}\right\}$ [5]. For the first bound, the AWGN at the relay is ignored and the distribution of $\gamma_{\text {end }}$ is replaced by the distribution of the half harmonic mean of $\gamma_{1}$ and $\gamma_{2}$. Based on this approximate distribution for $\gamma_{\mathrm{end}}$, a number of analyses have been performed [2], [6]-[10]. For the second bound, it is assumed that $\gamma_{1}$ and $\gamma_{2}$ are non-symmetric and the distribution of $\gamma_{\text {end }}$ is replaced by the distribution of the minimum between $\gamma_{1}$ and $\gamma_{2}$. This bound is usually exploited to analyze the average symbol error probability (ASEP) [11], [12], which has the intuitive meaning that the ASEP of the whole link (source-relaydestination) is dominated by the worst link between the source-torelay and relay-to-destination channels. Recently, the authors of [13] derive the exact probability density function (PDF) of the end-to-end SNR in a single-integral form, considering multi-hop CSI-assisted AF relaying scenario over general Nakagami fading channels.

Although the above bounds seem reasonable at high SNR, they are loose in the low and medium SNR regions. In this paper, the exact cumulative density function (CDF) of the end-to-end SNR of CSI-assisted dual-hop AF relaying is derived in an analytical form, considering transmission over arbitrary Nakagami- $m$ fading channels. In particular, with novel applications of bivariate $G$-function and $H$-function, an analytical CDF expression for the case with $m$ taking non-integer values is derived. To the best of the authors' knowledge, this is the first reported exact analytical CDF of the end-to-end SNR in CSI-assisted relaying systems with non-integer Nakagami- $m$ fading parameters, other than the exact single-integral solution in [13]. The obtained CDF is then applied to evaluate outage probability of the system under study. Monte Carlo simulations on outage probability are performed to illustrate the accuracy of our analytical results regardless of the SNR values and, in particular, our analytical results outperform the previously reported upper bounds in the low and medium SNR regions. The novel application of these two special functions are shown to be powerful tools in analyzing the performance of wireless systems in general and relaying techniques in particular.

\section{CDF OF THE END-TO-END SNR}

For a CSI-assisted dual-hop AF relaying system, the end-to-end SNR from the source to the destination was shown to be exactly given by [1]

$$
\gamma_{\mathrm{end}}=\frac{\gamma_{1} \gamma_{2}}{\gamma_{1}+\gamma_{2}+1},
$$

where $\gamma_{1}$ and $\gamma_{2}$ refer to the instantaneous SNRs at the first and second hops, respectively.

Assuming that the channels at two consecutive hops are subject to Nakagami- $m$ fading, the probability density function (PDF) of $\gamma_{i}$ in (1), where $i=1,2$, is expressed as [14, Eq.(2.21)]

$$
f_{\gamma_{i}}\left(\gamma_{i}\right)=\frac{m_{i}^{m_{i}}}{\Gamma\left(m_{i}\right) \bar{\gamma}_{i}^{m_{i}}} \gamma_{i}^{m_{i}-1} \exp \left(-\frac{m_{i}}{\bar{\gamma}_{i}} \gamma_{i}\right), \quad i=1,2
$$


where $m_{i}$ denotes the Nakagami fading parameter at the $i^{\text {th }}$ hop, $\Gamma($.$) stands for the Gamma function [15, Eq.(8.310)],$ $\bar{\gamma}_{1} \triangleq \mathbb{E}\left\{\left|h_{S R}\right|^{2}\right\} E_{S} / \sigma_{R}^{2}=m_{1}\left(m_{1}+1\right) E_{S} / \sigma_{R}^{2}$, and $\bar{\gamma}_{2} \triangleq$ $\mathbb{E}\left\{\left|h_{R D}\right|^{2}\right\} E_{S} / \sigma_{D}^{2}=m_{2}\left(m_{2}+1\right) E_{S} / \sigma_{D}^{2}$ with $\mathbb{E}\{$.$\} being the$ statistical expectation operator. Also, the CDF of $\gamma_{i}$ is given by [2]

$$
F_{\gamma_{i}}\left(\gamma_{i}\right)=1-\frac{1}{\Gamma\left(m_{i}\right)} \Gamma\left(m_{i}, \frac{m_{i}}{\bar{\gamma}_{i}} \gamma_{i}\right), \quad i=1,2
$$

where $\Gamma(.,$.$) stands for the upper incomplete Gamma function [15,$ Eq.(8.350.2)]. Finally, by integrating the conditional CDF of $\gamma_{\mathrm{end}}$ with respect to $\gamma_{1}$ over the PDF of $\gamma_{1}$, the CDF of $\gamma_{\text {end }}$ is given by

$$
\begin{aligned}
F_{\gamma_{\text {end }}}(\gamma)= & 1-\frac{C_{1}}{\Gamma\left(m_{2}\right)} \int_{0}^{\infty} \Gamma\left(m_{2}, \frac{m_{2} \gamma}{\bar{\gamma}_{2}}\left(1+\frac{\gamma+1}{x}\right)\right) \\
& \times(x+\gamma)^{m_{1}-1} \exp \left(-\frac{m_{1}}{\bar{\gamma}_{1}}(x+\gamma)\right) \mathrm{d} x
\end{aligned}
$$

where $C_{1} \triangleq m_{1}^{m_{1}} /\left(\Gamma\left(m_{1}\right) \bar{\gamma}_{1}^{m_{1}}\right)$. The integral term in (4) cannot be calculated directly, since the incomplete Gamma function is involved. In order to proceed, two different series expansions of the incomplete Gamma function are exploited and thus two different cases are discussed in the following, depending on the values of the fading parameters $m_{1}$ and $m_{2}$.

\section{A. Scheme with integer values for $m_{1}$ and $m_{2}$}

When $m_{2}$ takes integer values, the expansion of the incomplete Gamma function in (4) is a finite series [15, Eq.(8.352.7)] and thus this scheme can be easily analyzed. For the completeness of exposition, the CDF of the end-to-end SNR is reproduced and it is given by [3]

$$
\begin{aligned}
F_{\gamma_{\text {end }}}(\gamma) & \\
= & 1-2 C_{1} \sum_{n=0}^{m_{2}-1} \frac{1}{n !}\left(\frac{m_{2}}{\bar{\gamma}_{2}}\right)^{n} \exp \left[-\left(\frac{m_{1}}{\bar{\gamma}_{1}}+\frac{m_{2}}{\bar{\gamma}_{2}}\right) \gamma\right] \\
& \times \sum_{p=0}^{m_{1}-1} \sum_{q=0}^{n}\left(\begin{array}{c}
m_{1}-1 \\
p
\end{array}\right)\left(\begin{array}{c}
n \\
q
\end{array}\right) \gamma^{m_{1}+n-\frac{v_{1}}{2}} \\
& \times(\gamma+1)^{\frac{v_{1}}{2}}\left(\frac{m_{2} \bar{\gamma}_{1}}{m_{1} \bar{\gamma}_{2}}\right)^{\frac{v_{2}}{2}} K_{v_{2}}(2 \sqrt{\tilde{\gamma}})
\end{aligned}
$$

where $\left(\begin{array}{l}n \\ k\end{array}\right)$ denotes the binomial coefficient, $K_{v}(x)$ is the $v^{\text {th }}$-order modified Bessel function of the second kind [15, Eq.(8.432.6)], $v_{1} \triangleq$ $p+q+1, v_{2} \triangleq p-q+1$, and $\tilde{\gamma} \triangleq \frac{m_{1} m_{2} \gamma(\gamma+1)}{\bar{\gamma}_{1} \bar{\gamma}_{2}}$.

It is known that the Nakagami- $m$ fading reduces to the Rayleigh fading when $m_{1}=m_{2}=1$. Accordingly, putting $m_{1}=m_{2}=1$ into (5) reduces it to the result previously reported in [16, Eq.(2)].

\section{B. Scheme with non-integer values for $m_{1}$ and $m_{2}$}

When $m_{2}$ takes on non-integer values, the expansion of the incomplete Gamma function in (4) is an infinite series [15, Eq.(8.354.2)]. Substituting the infinite series into (4) and performing some algebraic manipulations yield (6) at the top of the next page.

Although the infinite series representation for the incomplete Gamma function is involved, this series is absolutely convergent for $m_{2} \geq 0.5$ and converges rapidly because of the factorial term $n$ ! in the denominator. Moreover, the integral term $I_{1}$ in (6) can be calculated as [15, Eq.(8.350.2)]

$$
\begin{aligned}
I_{1} & =C_{1}\left(\frac{\bar{\gamma}_{1}}{m_{1}}\right)^{m_{1}} \int_{\frac{m_{1}}{\bar{\gamma}_{1}} \gamma}^{\infty} x^{m_{1}-1} \exp (-x) \mathrm{d} x \\
& =\frac{1}{\Gamma\left(m_{1}\right)} \Gamma\left(m_{1}, \frac{m_{1}}{\bar{\gamma}_{1}} \gamma\right) .
\end{aligned}
$$

For the integral term $I_{2}$ in (6), the Newton's generalized binomial theorem $(1+x)^{m}=\sum_{n=0}^{\infty}\left(\begin{array}{c}m \\ n\end{array}\right) x^{n}$ cannot be applied, since the infinite series on the right-hand side converges only for $|x|<1$ $[17$, p.28]. Clearly, this condition is not satisfied for the binomials $\left(1+\frac{\gamma}{x}\right)^{m_{1}-1}$ and $\left(1+\frac{\gamma+1}{x}\right)^{m_{2}+n}$ in (6), where $0<x$ and $\gamma<\infty$. Therefore, how to calculate $I_{2}$ becomes challenging, which explains why no analytical CDF for $\gamma_{\text {end }}$ has been reported in the open literature till now. In the sequel, we exploit the Fox's $H$-function and the generalized Laplace transform of the product of two $H$-functions to tackle this problem, such that an analytical expression for $I_{2}$ is obtained.

From the definition of $I_{2}$ shown in (6), it can be reformulated as (8) in the middle of the next page, where $\tilde{m}_{1} \triangleq\left\lceil m_{1}\right\rceil-m_{1}$ and $\tilde{m}_{2} \triangleq\left\lceil m_{2}\right\rceil-m_{2}$ with $\lceil$.$\rceil being the integer ceiling operator,$ and the binomial expansion was exploited to reach (8). Applying the equality $(1+x)^{-\alpha}=\frac{1}{\Gamma(\alpha)} H_{1,1}^{1,1}\left[x \mid \begin{array}{c}(1-\alpha, 1) \\ (0,1)\end{array}\right]$ with $\alpha \geq 0$ [18, p.152] to (8), where $H[x \mid$.$] is the Fox's H$-function [18], we can express $I_{2}$ as (9) in the middle of the next page. Then, using the generalized Laplace transform of the product of two $H$ functions $^{1}$ [18, Eq.(2.6.2)], we obtain (10)-(11) in the middle of the next page, where [20, Eq.(6.4.1)] was exploited to arrive at (11) with $\mathrm{H}_{1}$ denoting the bivariate $H$-function and $\mathrm{G}_{1}$ being the bivariate $G$-function [19], which are given by

$$
\mathrm{H}_{1} \triangleq H_{1,[1: 1], 0,[1: 1]}^{1,1,1,1,1}\left[\begin{array}{c|c}
\frac{\bar{\gamma}_{1}}{m_{1} \gamma} & \left(1+p+q-m_{2}-n, 1\right) \\
\frac{\bar{\gamma}_{1}}{m_{1}(\gamma+1)} & (0,1) ;(0,1)
\end{array}\right]
$$

and

$$
\mathrm{G}_{1} \triangleq G_{1,[1: 1], 0,[1: 1]}^{1,1,1,1}\left[\begin{array}{c|c}
\bar{\gamma}_{1} & 1+p+q-m_{2}-n \\
\bar{m}_{1} \gamma & 1-\tilde{m}_{1} ; 1-\tilde{m}_{2} \\
\frac{\bar{\gamma}_{1}}{m_{1}(\gamma+1)} & - \\
0 ; 0
\end{array}\right],
$$

respectively.

Finally, substituting (7), (11), and $C_{1}$ into (6) and performing some algebraic manipulations, we obtain the CDF of the end-to-end SNR of dual-hop CSI-assisted relaying systems as (14) in the middle of the next page, where $C_{2} \triangleq 1 /\left(\Gamma\left(m_{1}\right) \Gamma\left(m_{2}\right) \Gamma\left(\tilde{m}_{1}\right) \Gamma\left(\tilde{m}_{2}\right)\right)$.

The infinite series in (14) is absolutely convergent. This is demonstrated as follows. Firstly, the bivariate $G$-function $G_{p,\left[t_{1}: t_{2}\right], s,\left[q_{1}: q_{2}\right]}^{n, v_{1}, v_{2}, w_{1}, w_{2}}\left[\begin{array}{l}x \\ y\end{array} \mid.\right]$ is defined in terms of double Mellin-Barnes type integrals, and it converges if the following conditions are satisfied [21, p.62]:

$$
\begin{gathered}
p+q_{1}+s+t_{1}<2\left(n+v_{1}+w_{1}\right), \\
p+q_{2}+s+t_{2}<2\left(n+v_{2}+w_{2}\right), \\
|\arg (x)|<\pi\left[n+v_{1}+w_{1}-\left(p+q_{1}+s+t_{1}\right) / 2\right], \\
|\arg (y)|<\pi\left[n+v_{2}+w_{2}-\left(p+q_{2}+s+t_{2}\right) / 2\right] .
\end{gathered}
$$

It is easy to show that the parameters of the $G$-function in (13) satisfy these sufficient conditions above and, therefore, the $G$-function converges in the sense of finite value. Secondly, it is clear that the infinite series in (14) is an alternating series and thus, by use of the Leibnitz's test [17, Theorem 1.5], it is known that this infinite series is conditionally convergent because of the factorial term $n$ ! in the denominator of the summand. Furthermore, applying the ratio test to the associated series of positive terms yields the infinite series in (14) to be absolutely convergent.

${ }^{1}$ There are several conditions need to be satisfied. They were carefully checked but omitted here due to space limitation. 


$$
\begin{aligned}
F_{\gamma_{\text {end }}}(\gamma)= & 1-\underbrace{C_{1} \int_{0}^{\infty}(x+\gamma)^{m_{1}-1} \exp \left(-\frac{m_{1}}{\bar{\gamma}_{1}}(x+\gamma)\right)}_{I_{1}} \mathrm{~d} x+\frac{C_{1}}{\Gamma\left(m_{2}\right)} \sum_{n=0}^{\infty} \frac{(-1)^{n}}{n !\left(m_{2}+n\right)}\left(\frac{m_{2}}{\bar{\gamma}_{2}} \gamma\right)^{m_{2}+n} \\
& \times \underbrace{\int_{0}^{\infty}(x+\gamma)^{m_{1}-1}\left(1+\frac{\gamma+1}{x}\right)^{m_{2}+n} \exp \left(-\frac{m_{1}}{\bar{\gamma}_{1}}(x+\gamma)\right) \mathrm{d} x}_{I_{2}}
\end{aligned}
$$

$$
\begin{aligned}
I_{2}= & \gamma^{m_{1}-1}(\gamma+1)^{m_{2}+n} \exp \left(-\frac{m_{1}}{\bar{\gamma}_{1}} \gamma\right) \int_{0}^{\infty} x^{-\left(m_{2}+n\right)} \exp \left(-\frac{m_{1}}{\bar{\gamma}_{1}} x\right)\left(1+\frac{x}{\gamma}\right)^{m_{1}-1}\left(1+\frac{x}{\gamma+1}\right)^{m_{2}+n} \mathrm{~d} x \\
= & \gamma^{m_{1}-1}(\gamma+1)^{m_{2}+n} \exp \left(-\frac{m_{1}}{\bar{\gamma}_{1}} \gamma\right) \int_{0}^{\infty} x^{-\left(m_{2}+n\right)} \exp \left(-\frac{m_{1}}{\bar{\gamma}_{1}} x\right)\left(1+\frac{x}{\gamma}\right)^{\left\lceil m_{1}-1\right\rceil-\tilde{m}_{1}}\left(1+\frac{x}{\gamma+1}\right)^{\left\lceil m_{2}+n\right\rceil-\tilde{m}_{2}} \mathrm{~d} x \\
= & \exp \left(-\frac{m_{1}}{\bar{\gamma}_{1}} \gamma\right) \sum_{p=0}^{\left\lceil m_{1}-1\right\rceil} \sum_{q=0}^{\left\lceil m_{2}+n\right\rceil}\left(\begin{array}{c}
\left\lceil m_{1}-1\right\rceil \\
p
\end{array}\right)\left(\begin{array}{c}
\left\lceil m_{2}+n\right\rceil \\
q
\end{array}\right) \gamma^{m_{1}-p-1}(\gamma+1)^{m_{2}+n-q} \\
& \times \int_{0}^{\infty} x^{p+q-\left(m_{2}+n\right)} \exp \left(-\frac{m_{1}}{\bar{\gamma}_{1}} x\right)\left(1+\frac{x}{\gamma}\right)^{-\tilde{m}_{1}}\left(1+\frac{x}{\gamma+1}\right)^{-\tilde{m}_{2}} \mathrm{~d} x .
\end{aligned}
$$

$$
\begin{aligned}
I_{2}= & \exp \left(-\frac{m_{1}}{\bar{\gamma}_{1}} \gamma\right) \sum_{p=0}^{\left\lceil m_{1}-1\right\rceil} \sum_{q=0}^{\left\lceil m_{2}+n\right\rceil}\left(\begin{array}{c}
\left\lceil m_{1}-1\right\rceil \\
p
\end{array}\right)\left(\begin{array}{c}
\left\lceil m_{2}+n\right\rceil \\
q
\end{array}\right) \gamma^{m_{1}-p-1}(\gamma+1)^{m_{2}+n-q} \\
& \times \frac{1}{\Gamma\left(\tilde{m}_{1}\right) \Gamma\left(\tilde{m}_{2}\right)} \int_{0}^{\infty} x^{p+q-\left(m_{2}+n\right)} \exp \left(-\frac{m_{1}}{\bar{\gamma}_{1}} x\right) H_{1,1}^{1,1}\left[\frac{x}{\gamma} \mid \begin{array}{c}
\left(1-\tilde{m}_{1}, 1\right) \\
(0,1)
\end{array}\right] H_{1,1}^{1,1}\left[\frac{x}{\gamma+1} \mid \begin{array}{c}
\left(1-\tilde{m}_{2}, 1\right) \\
(0,1)
\end{array}\right] \mathrm{d} x .
\end{aligned}
$$

$$
\begin{aligned}
I_{2} & =\frac{\exp \left(-\frac{m_{1}}{\bar{\gamma}_{1}} \gamma\right)}{\Gamma\left(\tilde{m}_{1}\right) \Gamma\left(\tilde{m}_{2}\right)} \sum_{p=0}^{\left\lceil m_{1}-1\right\rceil} \sum_{q=0}^{\left\lceil m_{2}+n\right\rceil}\left(\begin{array}{c}
\left\lceil m_{1}-1\right\rceil \\
p
\end{array}\right)\left(\begin{array}{c}
\left\lceil m_{2}+n\right\rceil \\
q
\end{array}\right) \gamma^{m_{1}-p-1}(\gamma+1)^{m_{2}+n-q}\left(\frac{m_{1}}{\bar{\gamma}_{1}}\right)^{m_{2}+n-p-q-1} \mathrm{H}_{1} \\
& =\frac{\exp \left(-\frac{m_{1}}{\bar{\gamma}_{1}} \gamma\right)}{\Gamma\left(\tilde{m}_{1}\right) \Gamma\left(\tilde{m}_{2}\right)} \sum_{p=0}^{\left\lceil m_{1}-1\right\rceil} \sum_{q=0}^{\left\lceil m_{2}+n\right\rceil}\left(\begin{array}{c}
\left\lceil m_{1}-1\right\rceil \\
p
\end{array}\right)\left(\begin{array}{c}
\left\lceil m_{2}+n\right\rceil \\
q
\end{array}\right) \gamma^{m_{1}-p-1}(\gamma+1)^{m_{2}+n-q}\left(\frac{m_{1}}{\bar{\gamma}_{1}}\right)^{m_{2}+n-p-q-1} \mathrm{G}_{1}
\end{aligned}
$$

$$
\begin{aligned}
F_{\gamma_{\text {end }}}(\gamma)= & 1-\frac{1}{\Gamma\left(m_{1}\right)} \Gamma\left(m_{1}, \frac{m_{1}}{\bar{\gamma}_{1}} \gamma\right)+C_{2} \exp \left(-\frac{m_{1}}{\bar{\gamma}_{1}} \gamma\right) \sum_{n=0}^{\infty} \frac{(-1)^{n}}{n !\left(m_{2}+n\right)}\left(\frac{m_{2}}{\bar{\gamma}_{2}} \gamma\right)^{m_{2}+n} \\
& \times \sum_{p=0}^{\left\lceil m_{1}-1\right\rceil} \sum_{q=0}^{\left\lceil m_{2}+n\right\rceil}\left(\begin{array}{c}
\left\lceil m_{1}-1\right\rceil \\
p
\end{array}\right)\left(\begin{array}{c}
\left\lceil m_{2}+n\right\rceil \\
q
\end{array}\right)\left(\frac{m_{1}}{\bar{\gamma}_{1}} \gamma\right)^{m_{1}-p-1}\left(\frac{m_{1}}{\bar{\gamma}_{1}}(\gamma+1)\right)^{m_{2}+n-q} \mathrm{G}_{1}
\end{aligned}
$$

Note that the bivariate $G$-function cannot be directly computed by popular mathematical softwares such as Matlab and Mathematica. In general, it has to be computed by its definition in terms of the double Mellin-Barnes type integrals [19], such as the Mathematica algorithm recently developed in [22]. Unfortunately, this definition is non-analytical and computationally intensive. In the following, we develop a general analytical expression to compute the bivariate $G$ function in the form of (13), which can also be applied to address other theoretical problems.

Since the convergence of the bivariate $G$-function in the form of (13) is guaranteed as per (15)-(18), according to [19, Eq.(2.3)], it can be expanded as (19)-(20) at the top of the next page, where in (19) the symbol $(a)_{b}=\Gamma(a+b) / \Gamma(a)$ denotes the Pochhammer operator, and we used the symbolic operators proposed in [23] to derive (20), which is a single series of the product of two generalized hypergeometric functions [15, Eq.(9.14.1)]. Note that the parameters $a, 1-b_{1}$, and $1-b_{2}$ cannot take negative integers since Gamma function is involved in (19)-(20), which is satisfied when non-integer $m_{1}$ and $m_{2}$ are applied to (13). Although it seems complicated, (20) involves only common special functions and it can be easily evaluated in a numerical way. The accuracy of (20) is corroborated by simulation results in the next section.

Remark II.1. (The PDF of the end-to-end SNR) After obtaining the CDF of the end-to-end SNR of CSI-assisted relaying over arbitrary Nakagami-m fading channels, its corresponding PDF can be readily obtained by taking the derivative of $F_{\gamma_{\text {end }}}(\gamma)$ with respect to $\gamma$. More specifically, the derivative of $K_{v}(\gamma)$ in (5) with respect to $\gamma$ can be obtained by using [15, Eq.(8.486.12)]. On the other hand, as a special case of bivariate $H$-function [20, Eq.(6.4.1)], the derivative of $\mathrm{G}_{1}$ in (14) can be obtained by exploiting the derivative of bivariate $H$-function shown in [20, Eq.(6.5.7)]. 


$$
\begin{aligned}
G_{1,[1: 1], 0,[1: 1]}^{1,1,1,1,1}\left[\begin{array}{c}
x \\
b_{1} ; b_{2} \\
- \\
0 ; 0
\end{array}\right]= & \Gamma(a) \Gamma\left(1-b_{1}\right) \Gamma\left(1-b_{2}\right) \sum_{p=0}^{\infty} \sum_{q=0}^{\infty} \frac{1}{p ! q !}(a)_{p+q}\left(1-b_{1}\right)_{p}\left(1-b_{2}\right)_{q}(-x)^{p}(-y)^{q} \\
= & \Gamma(a) \Gamma\left(1-b_{1}\right) \Gamma\left(1-b_{2}\right) \sum_{r=0}^{\infty} \frac{1}{r !}(a)_{r}\left(1-b_{1}\right)_{r}\left(1-b_{2}\right)_{r} \\
& \times x^{r} y^{r}{ }_{2} F_{0}\left(a+r, 1-b_{1}+r ;-;-x\right){ }_{2} F_{0}\left(a+r, 1-b_{2}+r ;-;-y\right)
\end{aligned}
$$

\section{Outage Probability}

Once the CDF and PDF of the end-to-end SNR have been obtained, they can be widely applied to evaluate the system performance in terms of different performance metrics. Based on the CDF, for example, the outage probability, outage capacity, and codeword error probability can be analytically obtained. On the other hand, by exploiting the PDF, the ergodic capacity and the output statistics such as the moments of the output SNR can be numerically evaluated. Herein, due to page limitation, we only demonstrate the accuracy of our main result (14) using outage probability. In all simulations, without loss of generality, the variances of the AWGN at the relay and at the destination are assumed to be identical, that is, $\sigma_{R}^{2}=\sigma_{D}^{2}$. Furthermore, the values of (14) are computed with the first 9 terms of the infinite series, in which the function $G_{1}$ is also computed with its first 9 terms as per (20). Further test results show that, when more than 9 terms from the infinite series in (20) are involved, both the proposed method and that in [22] yield almost the same output.

Outage probability, $P\left(\gamma_{\mathrm{th}}\right)$, is defined as the probability that the instantaneous output SNR falls below a pre-defined threshold $\gamma_{\mathrm{th}}$. Hence, evaluating the CDF (5) or (14) at $\gamma_{\text {th }}$, we obtain

$$
P\left(\gamma_{\mathrm{th}}\right)=\operatorname{Pr}\left\{\gamma_{\mathrm{end}}<\gamma_{\mathrm{th}}\right\}=F_{\gamma_{\mathrm{end}}}\left(\gamma_{\mathrm{th}}\right) .
$$

For comparison purposes, the outage probability based on the two upper bounds discussed in Section I are also reproduced here. For the first bound $\gamma_{\text {end }}<\frac{\gamma_{1} \gamma_{2}}{\gamma_{1}+\gamma_{2}}$ with the symmetric fading shape factors $m_{1}=m_{2}=m$, the outage probability is given by [6, Eq.(18)]

$$
\begin{aligned}
P^{\mathrm{b} 1}\left(\gamma_{\mathrm{th}}\right)= & \frac{\sqrt{\pi}}{2^{2 m-3} \Gamma^{2}(m)}\left(\frac{m}{\bar{\gamma}} \gamma_{\mathrm{th}}\right) \\
& \times G_{2,3}^{2,1}\left[\frac{4 m}{\bar{\gamma}} \gamma_{\mathrm{th}} \mid \begin{array}{c}
(0, m-0.5) \\
(m-1,2 m-1,-1)
\end{array}\right]
\end{aligned}
$$

where the superscript $b_{1}$ of $P^{\mathrm{b} 1}\left(\gamma_{\mathrm{th}}\right)$ refers to the first bound, and $G[. \mid$.$] denotes the Meijer's G$-function [15, Eq.(9.301)]. For the non-symmetric case with non-integer values $m_{1} \neq m_{2}$, to the best of our knowledge, no result was ever reported.

For the second bound $\gamma_{\text {end }}<\min \left\{\gamma_{1}, \gamma_{2}\right\}$, the outage probability is clearly given by

$$
P^{\mathrm{b} 2}\left(\gamma_{\mathrm{th}}\right)=F_{\gamma_{1}}\left(\gamma_{\mathrm{th}}\right)+F_{\gamma_{2}}\left(\gamma_{\mathrm{th}}\right)-F_{\gamma_{1}}\left(\gamma_{\mathrm{th}}\right) F_{\gamma_{2}}\left(\gamma_{\mathrm{th}}\right),
$$

where the superscript $b_{2}$ of $P^{\mathrm{b} 2}\left(\gamma_{\mathrm{th}}\right)$ refers to the second bound.

Figure 1 shows the outage probability of the systems with nonsymmetric non-integer fading parameters $\left(m_{1}, m_{2}\right)=(4.3,1.5)$, and the threshold $\gamma_{\mathrm{th}}=0,5 \mathrm{~dB}$. It is observed that the analytical results based on (21) coincide perfectly with the simulation results whereas the second bound (23) yields a small gap at low SNR. Notice that, since the first bound (22) holds only in the symmetric fading case (i.e., the fading parameters $m_{1}=m_{2}=m$ ), it cannot be applied to this non-symmetric fading scenario $\left(m_{1} \neq m_{2}\right)$.

Figure 2 presents the outage probability with symmetric noninteger fading parameters $m_{1}=m_{2}=1.5$, and the threshold $\gamma_{\mathrm{th}}=0,5 \mathrm{~dB}$. It is observed that the bound (23) performs worst and it is very loose in the whole SNR region under consideration, since this bound is derived with the assumption that the SNRs at consecutive hops are non-symmetric. The first bound (22) performs a little better than the second bound (23) but it is still loose in the low SNR region, since the effect of AWGN is ignored. On the other hand, our analytical result in (21) is always consistent with the simulation results.

Note that the CDF expression of the non-integer case in (14) cannot reduce to that of the integer case in (5). This is because we exploited two exclusive series expansions of the incomplete Gamma functions $\Gamma(m, x)$ with respect to the integer and non-integer values of $m$ [15, Eqs.(8.352.7) \& (8.354.2)], respectively. However, when a noninteger value of $m$ closely approaches an integer value, these two expansions should have almost the same numerical value; in other words, the result of (14) should be almost the same as that of (5). This is illustrated in Fig. 3. This figure shows the outage probability of different non-integer fading scenarios, compared with the integer fading scenario where $\left(m_{1}, m_{2}\right)=(1,3)$. For the non-integer cases, the fading parameter $m_{2}$ at the second hop is set to $m_{2}=2.999$, which is almost identical to the integer case with $m_{2}=3$. On the other hand, the fading parameter $m_{1}$ at the first hop varies from the worst case $m_{1}=0.5$ to $m_{1}=0.9$ and finally $m_{1}=0.98$. It is observed that, the worst fading parameter $m_{1}=0.5$ results in the highest outage probability. When $m_{1}$ increases, the outage probability of non-integer cases decreases and it becomes closer and closer to that of the integer case. Also, the analytical results coincide perfectly with the simulation results. This demonstrates the effectiveness of our derivations.

Finally, comparing Fig. 1 with Fig. 2, we observe that the slopes of all curves are identical at high SNR. Moreover, the slopes of the curves in Fig. 3 improve with $m_{1}$. These observations are in agreement with the well-known result that the diversity order of dualhop AF relaying systems is given by $\min \left\{m_{1}, m_{2}\right\}$ [4], [24].

\section{CONCLUSION}

Due to the difficulty of mathematical derivation, analyzing the performance of CSI-assisted AF relaying transmission in an exact way is very challenging, especially when the transmission is performed over the general Nakagami- $m$ fading channels. In this paper, exact expression for the distribution function of the end-to-end SNR was derived. In particular, when $m$ takes non-integer values, the Fox's $H$-function, bivariate $H$-function and $G$-function were exploited. Simulation results of outage probability corroborated all analytical results and these special functions were shown to be efficient tools for system performance evaluation.

\section{REFERENCES}

[1] M. O. Hasna and M.-S. Alouini, "End-to-end performance of transmission systems with relays over Rayleigh-fading channels," IEEE Trans. Wireless Commun., vol. 2, no. 6, pp. 1126-1131, Nov. 2003. 


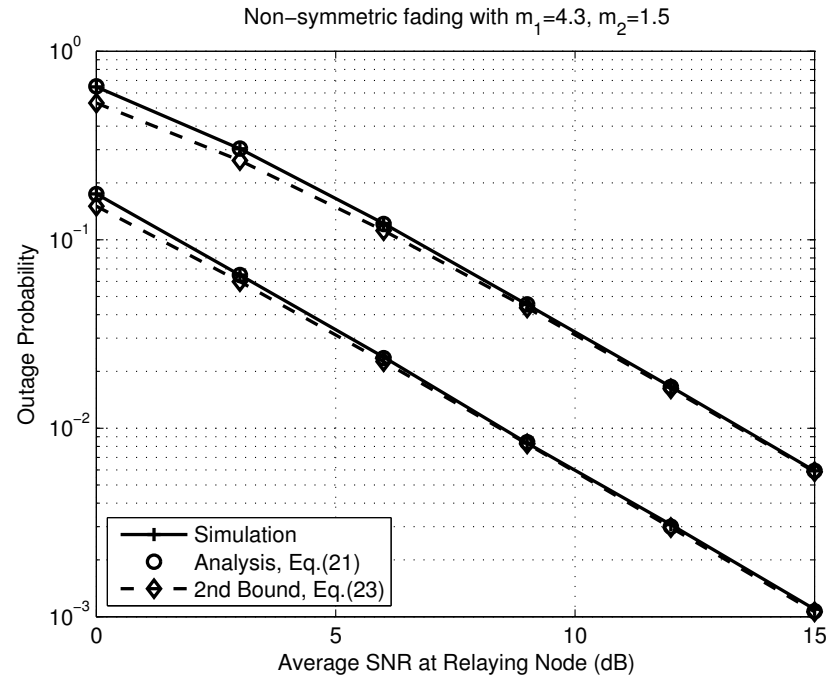

Fig. 1. Outage probability of dual-hop CSI-assisted AF relaying systems with non-symmetric non-integer Nakagami- $m$ fading parameters.

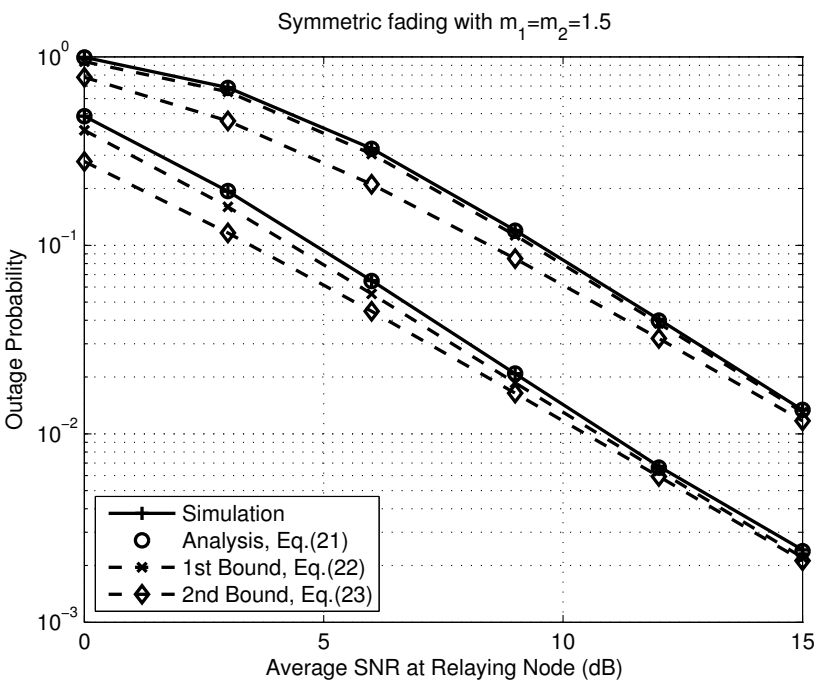

Fig. 2. Outage probability of dual-hop CSI-assisted AF relaying systems with symmetric non-integer Nakagami- $m$ fading parameters.

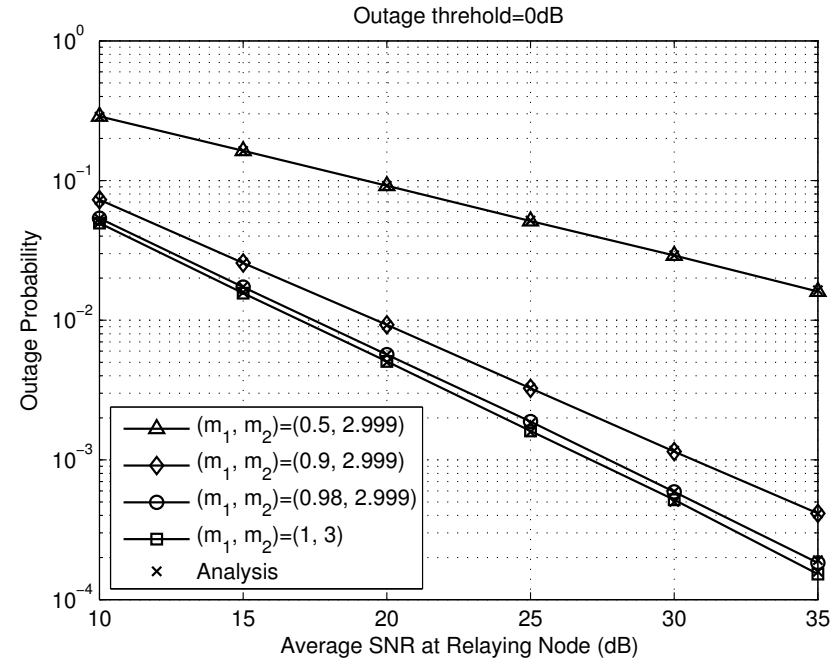

Fig. 3. Outage probability of dual-hop CSI-assisted AF relaying systems (non-integer versus integer fading parameters).
[2] D. B. da Costa and S. Aïssa, "Cooperative dual-hop relaying systems with beamforming over Nakagami- $m$ fading channels," IEEE Trans. Wireless Commun., vol. 8, no. 8, pp. 3950-3954, Aug. 2009.

[3] D. Senaratne and C. Tellambura, "Unified exact performance analysis of two-hop amplify-and-forward relaying in Nakagami fading," IEEE Trans. Veh. Technol., vol. 59, no. 3, pp. 1529-1534, Mar. 2010.

[4] M. Xia, C. Xing, Y.-C. Wu, and S. Aïssa, "Exact performance analysis of dual-hop semi-blind AF relaying over arbitrary Nakagami- $m$ fading channels," IEEE Trans. Wireless. Commun., vol. 10, no. 10, pp. 34493459, Oct. 2011.

[5] A. Bletsas, A. Khisti, D. P. Reed, and A. Lippman, "A simple cooperative diversity method based on network path selection," IEEE J. Sel. Areas Commun., vol. 24, no. 3, pp. 659-672, Mar. 2006.

[6] M. O. Hasna and M.-S. Alouini, "Harmonic mean and end-to-end performance of transmission systems with relays," IEEE Trans. Commun., vol. 52, no. 1, pp. 130-135, Jan. 2004.

[7] L.-L. Yang and H.-H. Chen, "Error probability of digital communications using relay diversity over Nakagami- $m$ fading channels," IEEE Trans. Wireless Commun, vol. 7, no. 5, pp. 1806-1811, May 2008.

[8] N. C. Beaulieu and Y. Chen, "An accurate approximation to the average error probability of cooperative diversity in Nakagami- $m$ fading," IEEE Trans. Wireless Commun., vol. 9, no. 9, pp. 2707-2711, Sep. 2010.

[9] V. Asghari, A. Maaref, and S. Aïssa, "Symbol error probability analysis for multihop relaying over Nakagami fading channels," in Proc. IEEE Wireless Commun. and Networking Conf. (WCNC'10), Sydney, Australia, pp. 1-5, Apr. 2010.

[10] M. Xia and S. Aïssa, "Moments based framework for performance analysis of one-way/two-way CSI-assisted AF relaying," IEEE J. Sel. Areas Commun., vol. 30, no. 8, Sep. 2012.

[11] D. B. da Costa and S. Aïssa, "End-to-end performance of dual-hop semi-blind relaying systems with partial relay selection," IEEE Trans. Wireless Commun., vol. 8, no. 8, pp. 4306-4315, Aug. 2009.

[12] S. Ikki and S. Aïssa, "Performance analysis of dual-hop relaying systems in the presence of co-channel interference," in Proc. IEEE Global Commun. Conf. (Globecom'10), pp. 1-5, Miami, FL, USA, Dec. 2010.

[13] N. C. Beaulieu and S. S. Soliman, "Exact analytical solution for endto-end SNR of multihop AF relaying systems," in Proc. IEEE Globecom'11 Workshop on Mobile Computing and Emerging Communication Networks, pp. 580-585, Houston, USA, Dec. 2011.

[14] M. K. Simon and M.-S. Alouini, Digital Communication over Fading Channels, 2nd Ed., John Wiley \& Sons Inc., 2005.

[15] I. S. Gradshteyn and I. M. Ryzhik, Table of Integrals, Series, and Products, 7th Ed., Academic Press, 2007.

[16] B. Barua, H. Q. Ngo, and H. Shin, "On the SEP of cooperative diversity with opportunistic relaying," IEEE Commun. Lett., vol. 12, no. 10, pp. 727-729, Oct. 2008.

[17] L. C. Andrews, Special Functions of Mathematics for Engineers, 2nd Ed., McGraw-Hill, Inc., 1992.

[18] A. M. Mathai and R. K. Saxena, The H-function with Applications in Statistics and Other Disciplines, Wiley Eastern, 1978.

[19] R. P. Agarwal, "An extension of Meijer's G-function", Proc. Nat. Inst. Sci. India, Part A, vol. 31, pp. 536-546, 1965.

[20] H. M. Srivastava, K. C. Gupta, and S. P. Goyal, The H-functions of One and Two Variables with Applications, New Delhi: South Asian Publishers, 1982.

[21] A. M. Mathai and R. K. Saxena, "Expansion of Meijer's $G$-function of two variables when the upper parameters differ by integers," Kyungpook Math. J., vol. 12, no. 1, pp. 61-68, June 1972.

[22] I. S. Ansari, S. Al-Ahmadi, F. Yilmaz, M.-S. Alouini, and H. Yanikomeroglu, "A new formula for the BER of binary modulations with dual-brance selection over gereralized- $K$ composite fading channels," IEEE Trans. Commun, vol. 59, no. 10, pp. 2654-2658, Oct. 2011.

[23] J. L. Burchnall and T. W. Chaundy, "Expansion of Appell's double hypergeometric functions," Quarterly J. Math. (Oxford), vol. 11, pp. 249270, Nov. 1940.

[24] Y. Li and S. Kishore, "Asymptotic analysis of amplify-and-forward relaying in Nakagami-fading environments," IEEE Trans. Wireless Commun., vol. 6, no. 12, pp. 4256-4262, Nov. 2007. 